\title{
The Influence of Microscope and Specimen Parameters on the Spatial Resolution of Transmission Kikuchi Diffraction
}

\author{
Glenn Sneddon ${ }^{1}$, Pat Trimby ${ }^{1,2}$, Julie Cairney ${ }^{1}$ \\ ${ }^{1}$ Australian Centre for Microscopy and Microanalysis, University of Sydney, Sydney, Australia \\ ${ }^{2}$ Oxford Instruments Nanoanalysis, High Wycombe, U.K.
}

Recently, there has been significant interest in using transmission Kikuchi diffraction (TKD) in scanning electron microscopes (SEM) for studying ultrafine-grained and nanocrystalline materials and nanostructures. While absolute spatial resolutions of down to 5-10 nm have been attained [1], how the achievable spatial resolution is affected by different experimental parameters is not well understood. Rice et al. [2] considered the effect of very high sample thicknesses on the spatial resolution, and demonstrated where the technique was superior to Electron Backscatter Diffraction (EBSD) but did not investigate the absolute limits of TKD. Wang et al. [3] also examined the impact of specimen thickness but factors such as specimen tilt or the material of the specimen being examined have yet to be considered. There is also a very limited understanding of the depth resolution of TKD, with existing experimental studies so far only examining which side of the sample the Kikuchi pattern is coming from [2]. These are potentially important factors that need to be considered to better understand and leverage the advantages and limitations of TKD.

For TKD, the depth resolution can be considered to be the distance sample surface between two points: the bottom surface of the specimen and the highest location of the final diffraction relevant event that noticeably contributes to the Kikuchi pattern [5]. In this study, the depth resolution was measured by using bilayered specimens consisting of a coarse-grained aluminium layer and a nanocrystalline layer of either platinum, copper or aluminium. The relative contributions of these layers was determined by comparing point patterns to patterns averaged over an area, at different nanocrystalline layer thicknesses. By averaging the patterns, only the pattern from the coarse layer is visible in those images. Figure 1 shows a comparison of these patterns for untilted coarse-grained aluminium/nanocrystalline copper samples at $30 \mathrm{keV}$ (all experiments for this work were carried out using a Carl Zeiss Ultra Plus field emission SEM equipped with an Oxford Instruments Aztec EBSD and TKD system). The thickness at which the nanocrystalline layer started to become dominant was 5, 15 and $50 \mathrm{~nm}$ in platinum, copper and aluminium respectively and the depths at which the coarse layered pattern was no longer visible in the Kikuchi patterns were 13, 33 and $80 \mathrm{~nm}$.

The absolute lateral resolution for TKD measures the lateral spread of the electrons contributing to a single Kikuchi pattern. However, the effective resolution obtained is generally better, with deconvolution of the Kikuchi patterns enabling overlapping patterns to be indexed to a single point. In this study, the absolute lateral resolution was measured by acquiring TKD linescans across a number of grain boundaries at different sample thicknesses, accelerating voltages and specimen backtilts. The Kikuchi patterns were correlated against a baseline pattern from each grain and a normalised correlation coefficient of 0.2 was used as a threshold value for the presence of a specific pattern, with absolute lateral resolution taken as the size of this overlapping region. An example of the type of normalised correlation plot is shown in Figure 2. It was found that sample thickness had the most significant impact on the lateral resolution with increasing sample thickness worsening the resolution. Increasing the specimen backtilt also caused a worsening of the resolution but changing the accelerating voltage did not seem to have a significant effect on the lateral resolution (although past a certain thickness, it did decrease the quality of the Kikuchi patterns). 
References:

[1] PW Trimby, Ultramicroscopy 120 (2012) p. 16-24.

[2] KP Rice, RR Keller and MP Stoykovich, Microsc. Today 23 (2015) p. 32-37.

[3] YZ Wang et al. J. Microsc. 264 (2016), p. 1-7

[4] GC Sneddon, PW Trimby and JM Cairney, Materials Science and Engineering R: Reports, 110 (2016), p. 1-12.

[5] The authors acknowledge the facilities and the scientific and technical assistance of the Australian Microscopy \& Microanalysis Facility at the Australian Centre for Microscopy \& Microanalysis at the University of Sydney

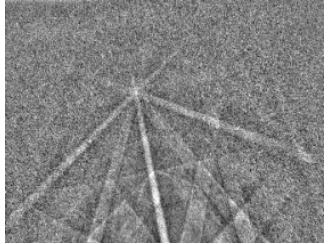

$7 \mathrm{~nm}$

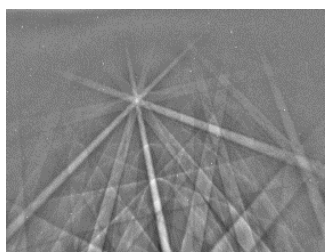

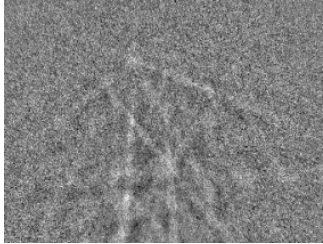

$14 \mathrm{~nm}$

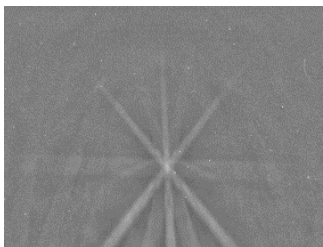

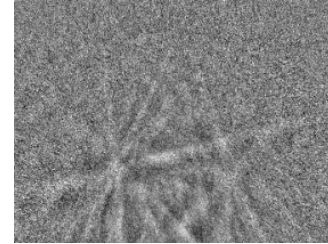

$19 \mathrm{~nm}$

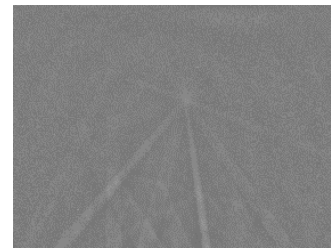

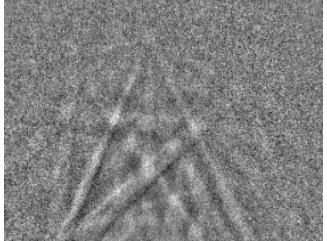

$27 \mathrm{~nm}$

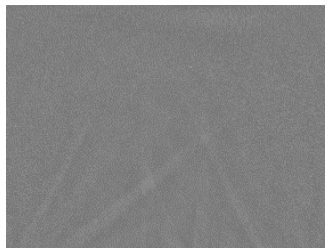

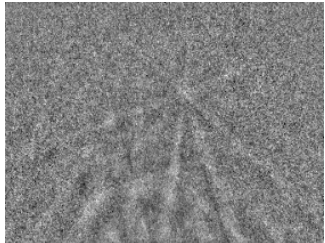

$33 \mathrm{~nm}$

Figure 1 Point patterns (top) and averaged map patterns (bottom) from bilayered coarse grained $\mathrm{Al} /$ nanocrystalline $\mathrm{Cu}$ samples, with different nanocrystalline layer thicknesses

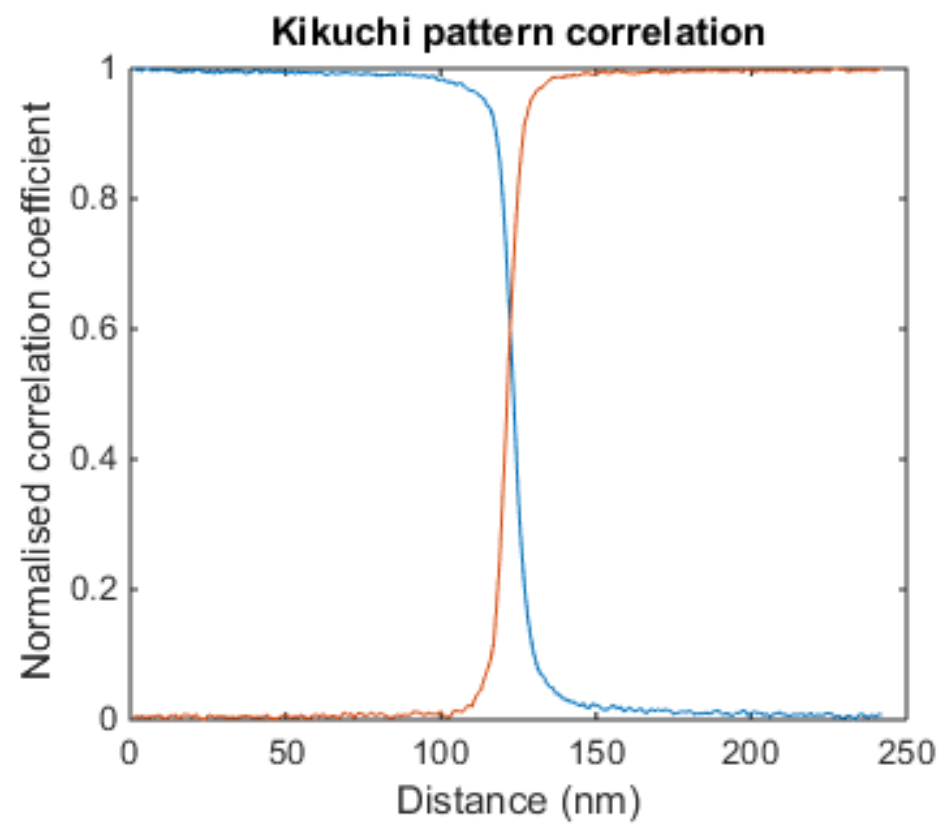

Figure 2 Example normalised correlation plot for a TKD linescan with Kikuchi patterns correlated to a reference pattern from each grain 